\title{
Material limitations of carbon-nanotube inertial balances: on the possibility of intrinsic yoctogram mass resolution at room temperature
}

\author{
Johannes Lischner and T.A. Arias \\ Laboratory of Atomic and Solid State Physics, Cornell University, Ithaca, New York 14853
}

\begin{abstract}
We present a theoretical study of the intrinsic quality factor of the fundamental flexural vibration in a carbon nanotube and its dependence on temperature, radius, length and tension. In particular, we examine three- and four-phonon decays of the fundamental flexural mode within quantized elasticity theory. This analysis reveals design principles for the construction of ultrasensitive nanotube mass sensors: under tensions close to the elastic limit, intrinsic losses allow for single yoctogram mass resolution at room temperature, while cooling opens the possibility of sub-yoctogram mass resolution.

PACS numbers: 63.22.Gh,62.25.-g,63.20.kg
\end{abstract}

Recently, much experimental effort has gone into fabricating nano-electromechanical systems (NEMS) which employ carbon nanotubes as mechanical resonators [1-4] The combination of small mass density and large mechanical stiffness makes carbon nanotube NEMS ideal candidates for ultrasensitive mass detectors which do not require disruptive ionization of the analyzed molecule: recent experiments achieved a mass resolution of $\sim 100 \mathrm{yg}$ $\left(1 \mathrm{yg}=10^{-24} \mathrm{~g}\right)$ [5, 6]. However, for isotopic or chemical identification of molecules adsorbed on the nanotube or real-time monitoring of chemical reactions yoctogram mass resolution is necessary.

The mass resolution of nanotube NEMS depends strongly on the quality factor of the lowest flexural mode, which imposes a lower bound on the frequency differences which can be resolved. The quality factors found in most experiments have been surprisingly low, not exceeding $Q \approx 2000$ [4, [5, 7]. Only very recently, Hüttel et al. 3] measured $Q \gtrsim 10^{5}$, but at milikelvin temperatures.

The intrinsic quality factor of a nanotube sets aside all extrinsic losses, such as defect or clamping losses, and sets an upper limit to the quality factor achievable in experiment. Both molecular dynamics simulations [8] and analytical approaches [9, 10] have been used to study the intrinsic quality factor theoretically, finding relatively low values similar to those in the experiments. However, simulations are limited to extremely short tubes, and the analytical approaches to date have either employed simplified phonon spectra [9] or focused solely on lowtemperature four-phonon decays of the flexural mode 10] using an analysis appropriate to extremely long tubes.

In this Letter, we present a theoretical analysis appropriate to the length of tubes and operating temperatures commonly found in experiments. We discover that application of tension drastically increases the intrinsic quality factor of nanotube oscillators and opens for the first time the theoretical possibility of single yoctogram mass resolution in such oscillators at room temperature, with further improvements possible with cooling. Indeed, the recent experiments of Wei et al. demonstrate a promising technique for controlling the tension in nanotubes [11].
For our analysis, we employ continuum elastic theory, which reliably describes long wavelength phonons in nanotubes, to study the decay of the lowest flexural mode due to phonon-phonon interactions, the most important source of intrinsic losses in semiconducting, and possibly also metallic, nanotubes 10]. Following the work of De Martino et al. 10] and Suzuura et al. 12], we describe a nanotube as a rolled-up two dimensional elastic sheet and expand the free energy in powers of the strain tensor $u_{i j}$ and the curvature tensor $S_{i j}$. Curvature contributions to the free energy are generally small, but need to be included in the quadratic part to describe optical phonons properly. Using the isotropy of the elastic sheet leads to

$$
\begin{aligned}
& \mathcal{U}_{2}=\frac{\lambda}{2}(\operatorname{Tr} u)^{2}+\mu \operatorname{Tr} u^{2}+\frac{k_{c}}{2}\left(\operatorname{Tr}\left(S-S_{0}\right)\right)^{2}, \\
& \mathcal{U}_{3}=\zeta_{1}(\operatorname{Tr} u)^{3}+\zeta_{2} \operatorname{Tr} u^{2} \operatorname{Tr} u,
\end{aligned}
$$

where $\mathcal{U}_{2}$ and $\mathcal{U}_{3}$ denote contributions to the energy density including quadratic and cubic powers of $u_{i j}$ and $S_{i j}$. Also, $\lambda$ and $\mu$ denote the Lamé moduli, $k_{c}$ the bending stiffness and $S_{0}$ the curvature tensor of the undeformed tube. $\zeta_{1}$ and $\zeta_{2}$ are anharmonic elastic constants.

Next, using the standard expressions for $u_{i j}$ and $S_{i j}$ in cylindrical coordinates [10, 12], we express the potential energy in terms of displacements $u_{n}$ with $n=x, y, z$ ( $x$ denotes the tangential direction, $y$ is along the tube axis and $z$ is the radial direction). $u_{i j}$ contains both linear and nonlinear contributions in $u_{n}$, which gives rise to two types of nonlinearities in the resulting Hamiltonian: material nonlinearities, described by the explicitly higher-order terms in Eq. (2), and geometric nonlinearities, where the lower order terms, such as terms in Eq. (11), contribute at higher orders due to higher order terms in the expansion of $u_{i j}$ in terms of the degrees of freedom $u_{n}$. Material nonlinearities are often suppressed by a factor of $k R$ ( $k$ being the phonon wave vector and $R$ the tube radius) compared to geometric nonlinearities [10] and can therefore be neglected for long wavelength phonons. However, when evaluating the three-phonon contribution to the quality factor, high energy phonons, whose wavelength is no longer comparable to the length 


\begin{tabular}{ccccc}
\hline \hline$\lambda\left[\mathrm{J} / \mathrm{m}^{2}\right]$ & $\mu\left[\mathrm{J} / \mathrm{m}^{2}\right]$ & $\zeta_{1}\left[\mathrm{~J} / \mathrm{m}^{2}\right]$ & $\zeta_{2}\left[\mathrm{~J} / \mathrm{m}^{2}\right]$ & $k_{c}[\mathrm{eV}]$ \\
\hline 59.57 & 147.94 & -145.17 & -387.93 & 1.46 \\
\hline \hline
\end{tabular}

TABLE I: Lamé moduli, anharmonic elastic constants of $\mathcal{U}_{3}$ and bending stiffness for semiconducting carbon nanotubes from $a b$ initio density-functional calculations.

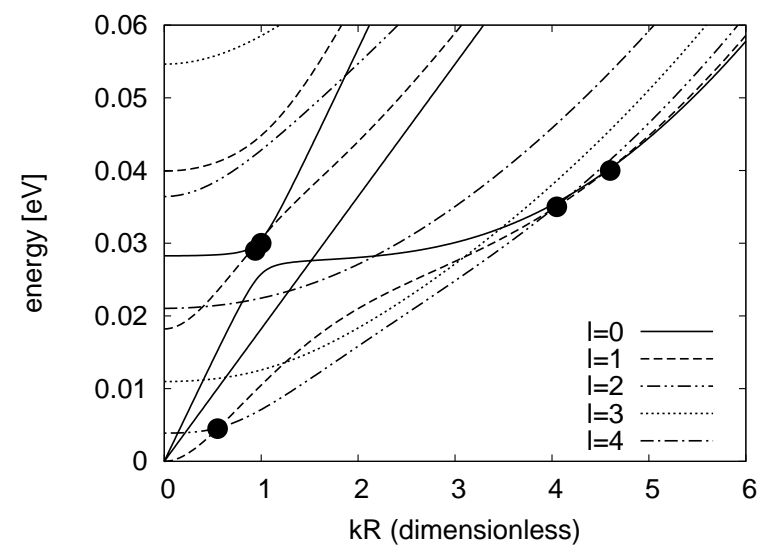

FIG. 1: Energy of phonons versus $k R$ for a $R=0.5 \mathrm{~nm}$ tube. The black dots denote phonon crossings, where three-phonon decays of the fundamental flexural mode are allowed.

of the tube, play a crucial role. We, therefore, also consider in this work the contribution of material nonlinearities in the three-phonon decay channel.

To determine accurate numerical values of the elastic constants in Eqs. (11) and (2), we evaluate total energies of strained graphene sheets and various semiconducting nanotubes within density-functional theory in the local density approximation [13] and fitted the results to the continuum theory described above. Table \ summarizes our results, which for the elastic constants of $\mathcal{U}_{2}$ are in good agreement with previous calculations [14, 15].

We next impose canonical commutation relations to quantize the resulting elastic theory and diagonalize the quadratic part of the Hamiltonian by expressing the displacement operator in terms of normal modes.

Figure 1 shows the resulting phonon dispersion curves for a tube with radius $R=0.5 \mathrm{~nm}$, which are in good agreement with force constant models [16] and ab initio calculations [15]. In particular, we find as the lowestfrequency long wavelength modes two degenerate flexural modes with angular momentum component $\ell= \pm 1$ along the tube axis and a quadratic dispersion relation

$$
\omega_{F}(k)=\frac{\hbar k^{2}}{2 m}+\mathcal{O}\left(k^{4}\right), \quad m=\frac{\hbar}{R} \sqrt{\frac{\rho_{M}(\lambda+2 \mu)}{8 \mu(\lambda+\mu)}},
$$

where $\omega_{F}$ denotes the angular frequency of the flexural phonon and $\rho_{M}=7.68 \times 10^{-7} \mathrm{~kg} / \mathrm{m}^{2}$ is the areal mass density of graphene. We also find a variety of low-lying optical phonons. Note that inclusion of curvature terms into Eq. (1) is crucial to obtain the correct optical phonon spectrum. In particular, the gap of the lowest optical branch vanishes if curvature terms are neglected.

Next, we employ conservation laws to analyze the possible decay mechanisms of the fundamental flexural mode. In addition to energy conservation, translational invariance along the tube axis imposes conservation of $k$. Rotational invariance around the tube axis imposes conservation of $\ell$. These conservation laws forbid the decay of the fundamental flexural mode in a three-phonon process which involves two other low-frequency modes [10]. If, however, the fundamental flexural mode, which has a wavelength comparable to the length of the tube, interacts with a high-energy phonon of momentum $k_{\mu}$, energy and momentum conservation enforce that the third phonon also has a high energy and momentum $k_{\nu} \approx k_{\mu}$. Because the flexural mode carries $\ell= \pm 1$, the angular momenta of the two high-energy phonons must differ by one. In sum, three-phonon decays of the fundamental flexural mode are only possible at crossings of two phonon bands whose angular momentum quantum numbers differ by one. Inspection of Fig. 1 reveals that very few such crossings for energies comparable and smaller than $k_{B} T \approx 24 \mathrm{meV}$ (at room temperature) exist.

To compute the three-phonon contribution to the quality factor, we evaluate the imaginary part of the Matsubara Green function obtained from the lowest order bubble diagram, which contains a sum over intermediate high-energy phonon momenta. Because the energy uncertainty resulting from the short lifetimes [17] of these modes is larger than the energy difference of phonons at neighboring allowed wave vectors of the finite-length tube, the sum can be converted into an integral. Then we use the energy-conserving $\delta$-function to reduce the integral into a sum over allowed crossings. Our final expression for the inverse quality factor resulting from the decay of a long wavelength flexural mode of wave vector $k$ in a three-phonon process involving two high-energy modes (labeled $\mu$ and $\nu$ ) at a phonon crossing at wave vector $k_{\times}$and angular frequency $\omega_{\times}$is

$$
Q_{3}^{-1}=\sum_{\times} g_{F \mu \nu}\left(k, k_{\times}, \ell_{\mu}\right) \frac{\beta \hbar^{2} n\left(\omega_{\times}\right)\left[n\left(\omega_{\times}\right)+1\right]}{R \omega_{F}(k) \omega_{\times}^{2}\left|v_{\mu}^{\times}-v_{\nu}^{\times}\right|},
$$

where $g_{F \mu \nu}$ is a complicated coupling function that depends on the polarization vectors of all three phonons and gives a complete description of both material and geometric nonlinearities. Also, $\sum_{\times}$denotes a sum over allowed crossings; $v_{\mu / \nu}^{\times}$are the phonon group velocities and $n(\omega)=1 /(\exp (\beta \hbar \omega)-1)$ is the Bose-Einstein factor with $\beta=1 /\left(k_{B} T\right)$ being the inverse thermal energy.

Experiments with doubly clamped tubes inevitably involve some amount of strain. Here we consider the case of positive strain, corresponding to some amount of ten- 
sion in the tube. To study the quality factor of such a strained tube, we expand the displacement around the new equilibrium value, taking into account the relaxation in the equilibrium radius. Evaluation of Eq. (2) at the strained equilibrium configuration leads to two categories of additional contributions to the quadratic hamiltonian: one set of terms is already present in $\mathcal{U}_{2}$ and can be absorbed into a redefinition of the linear elastic constants; the other set of terms gives the expected Hamiltonian for a string under tension, proportional to $\left(\partial_{y} u_{x}\right)^{2}+\left(\partial_{y} u_{z}\right)^{2}$ with a prefactor proportional to the tension.

Computationally, having obtained the phonon dispersions for a given radius, we numerically determine $k_{\times}$, $\omega_{\times}$and $v_{\mu / \nu}^{\times}$, which are needed to evaluate $g_{F \mu \nu}$ and ultimately the losses, for all relevant crossings. Our results indicate that geometric nonlinearities give the largest contribution to three-phonon losses, with material nonlinearities contributing only about one percent. Also, we find that the primary effect from the application of tension is to change the frequencies of the acoustic phonons, while the polarization vectors and the optical phonon frequencies change very little.

Figure 2 shows our results for the three-phonon contribution to the inverse quality factors for tubes of typical experimental radii as a function of inverse temperature. At low temperatures [right side of Fig. 2(a)], $Q_{3}^{-1}$ approaches zero exponentially, because the first BoseEinstein factor in Eq. (4) rapidly diminishes the occupation of the high-energy modes at the crossings, which are the modes responsible for the scattering. At higher temperatures, $T>100 \mathrm{~K}$, the modes associated with the relevant crossings are classically occupied and we find that $Q_{3}^{-1}$ is proportional to temperature.

Comparing the inverse quality factors of tubes of different radii in Fig. 2(a), we find that at low temperatures the tube with the smallest radius, $R=0.3 \mathrm{~nm}$, exhibits the lowest dissipation, while at high temperatures its losses are largest [see Fig. 2(b)].

To understand this nontrivial radius dependence of $Q_{3}$, we note that for $T<40 \mathrm{~K}$ the largest contribution to the losses comes from the crossing which is lowest in energy. The energy of this crossing of the flexural mode and the lowest optical mode is approximately equal to the gap of the optical mode given by $E_{g a p}=\sqrt{k_{c} /\left(5 \rho_{M}\right)} 6 \hbar / R^{2}$, which depends sensitively on the radius of the tube. Therefore, at low temperatures, the occupation of the high-energy modes at this crossing is much more strongly suppressed for tubes with smaller radii (and therefore higher optical frequency), which leads to smaller losses according to Eq. (4). At higher temperatures, the contributions from other crossings become important (see Fig. (1). We find that those contributions depend quite sensitively on $R$, which leads to the observed crossover behavior of the $R=0.3 \mathrm{~nm}$ tube.

Figure 3(a) shows the dependence of $Q_{3}$ on the length $L$ of the nanotube. In a tensionless tube (solid line),
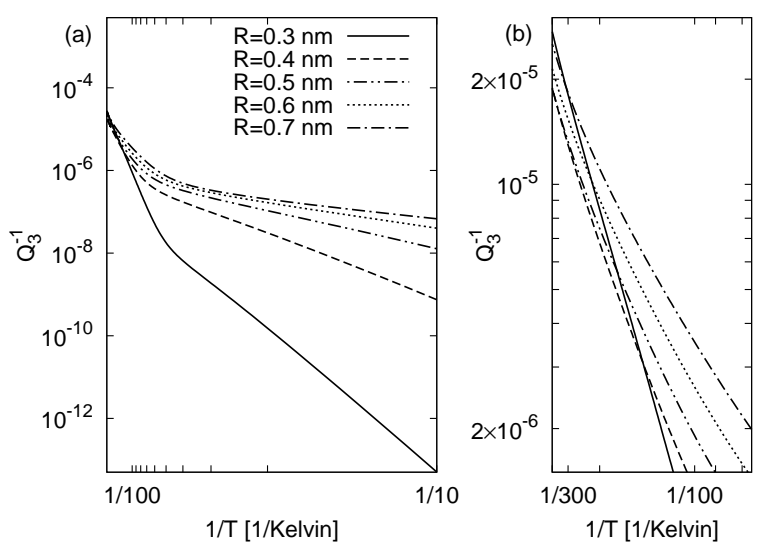

FIG. 2: Log-log plots of the temperature dependence of $Q_{3}$ for tubes of different radii and length $L=500 \mathrm{~nm}$.
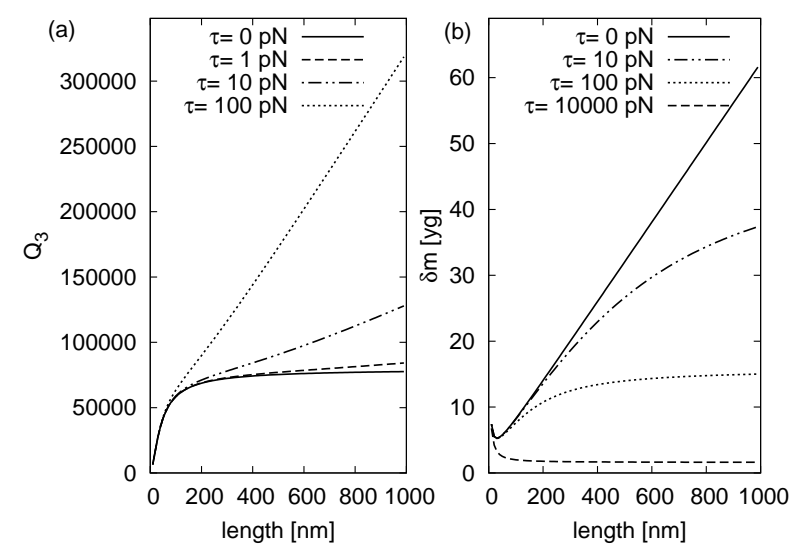

FIG. 3: (a) Length dependence of $Q_{3}$ for tubes with $R=$ $0.5 \mathrm{~nm}$ at $T=300 \mathrm{~K}$. (b) Length dependence of mass resolution $\delta m$ for tubes with $R=0.5 \mathrm{~nm}$ at $T=300 \mathrm{~K}$.

we find a remarkable cancellation between the length dependence of the coupling function, $g_{F \mu \nu} \propto k^{2}$ for small $k$, and the length dependence of $\omega_{F} \propto k^{2}$ in Eq. (4), resulting in a quality factor which is insensitive to tube length beyond $\sim 300 \mathrm{~nm}$. Strained tubes do not exhibit this cancellation because $\omega_{F}(k)$ is shifted by a constant proportional to the tension $\tau$ if the tension is small (dashed line) [18]. If $\tau$ is large, $\omega_{F}(k) \propto \sqrt{\tau} k$ and $Q_{3}$ becomes linear in $L$ for long tubes (dotted line) [18].

For tubes shorter than $300 \mathrm{~nm}$, Fig. 3(a) shows a significant length dependence of $Q_{3}$ even for tensionless tubes. In particular, in tubes with lengths of only a few nanometers $Q_{3}$ is reduced by more than an order of magnitude. This may be related to the small quality factors, $Q \approx 1500$, found by Jiang et al. [8], who model the decay of the fundamental flexural mode in a $3 \mathrm{~nm}$ long singly clamped tube via molecular dynamics simulations.

Next, we compare the magnitude of the computed intrinsic quality factor to experimental findings. At $T=300 \mathrm{~K}$, we find $Q_{3} \gtrsim 5 \times 10^{4}$, which is at least 
one order of magnitude larger than experimental results, $Q_{\text {exp }} \lesssim 2000$ [4, 7], suggesting that it is worthwhile to continue improving the control of losses in experiments. We find that the resulting (intrinsic) mass resolution, $\delta m=2 M / Q$, of a tensionless nanotube mass sensor depends sensitively on the tube length $L$ with a minimum of $\sim 5 \mathrm{yg}$ for very short tubes [Fig. 3(b)]. On the other hand, we find (i) that application of tension can reduce the mass resolution $\delta m$ to a single yoctogram if a tension close to the elastic limit, $\tau_{c} \approx 100 \mathrm{nN}$ [19], is applied and (ii) that $\delta m$ becomes independent of $L$ for long tubes, simplifying the design and fabrication of actual devices.

At lower temperatures, as discussed above, threephonon processes are exponentially suppressed due to the energy gap of the optical modes, and the resulting dissipation becomes much smaller than the experimental findings in the milikelvin range [3]. We, therefore, now move on to consider losses from four-phonon decays.

To estimate the role of four-phonon processes, we compute the leading order contribution, the fishbone diagram, due to a quartic coupling between four low-energy flexural modes. Following De Martino et al. [10] and our findings for the three-phonon case, we only take into account quartic geometric nonlinearities resulting from replacing $u_{i j}$ in Eq. (1) by its nonlinear part. The resulting expression for the four-phonon contribution to the quality factor contains a triple sum over intermediate phonon momenta and is given by 20 ]

$$
\begin{aligned}
Q_{4}^{-1}= & \sum_{q_{1} q_{2} q_{3}} \sum_{\xi_{1}, \xi_{2}, \xi_{3}= \pm} \frac{\xi_{1} \xi_{2} \xi_{3} \mathcal{D}\left(q_{1}, q_{2}, q_{3}, k\right) \eta / \pi}{\left(\omega_{F}(k)+\xi_{1} \omega_{1}+\xi_{2} \omega_{2}+\xi_{2} \omega_{3}\right)^{2}+\eta^{2}} \\
& \times \frac{n\left(\xi_{1} \omega_{1}\right) n\left(\xi_{2} \omega_{2}\right) n\left(\xi_{3} \omega_{3}\right)}{n\left(\xi_{1} \omega_{1}+\xi_{2} \omega_{2}+\xi_{3} \omega_{3}\right)},
\end{aligned}
$$

where $q_{i}$ denote intermediate momenta, $\omega_{i} \equiv \omega_{F}\left(q_{i}\right)$ and $\mathcal{D}$ denotes the coupling function. Here, $\eta$ is the inverse lifetime associated with the dressed phonon lines representing the actual phonons in the system with loss.

In evaluating Eq. (5), proper account of the finite length of the tube, which leads to a finite spacing of the allowed wave vectors, is of crucial importance. For long wavelength flexural phonons in tubes of experimental lengths, the energy uncertainty $\Delta E_{\eta}$ associated with the observed lifetimes is actually much smaller than the energy difference $\Delta E_{\Delta k}$ of phonons at neighboring wave vectors: Expressing $\eta$ in terms of the quality factor, $\eta=\omega_{F} /(2 \pi Q)$, we find $\Delta E_{\eta} / \Delta E_{\Delta k}=1 /(4 \pi Q)$, which is much smaller than unity at low temperatures [3]. Thus, the sums over intermediate momenta cannot be converted into integrals for the tubes in the experiments. Converting the sums into integrals, as De Martino et al. [10] do, is appropriate for much longer tubes, but leads to an underestimate of $Q_{4}$ due to inclusion of processes which are not present in the experiments.

To describe four-phonon decays in experimentally relevant nanotubes, we carry out numerically the discrete triple sum over intermediate momenta in Eq. (5) using the discrete frequencies of a finite-length doubly clamped beam and experimentally observed inverse lifetimes $\eta$. The result then gives the contribution to the observed losses from four-phonon processes. The resulting contributions to the quality factor are $Q_{4}=6.6 \times 10^{8}$ at $T=1 \mathrm{~K}$ and $Q_{4}=1.6 \times 10^{14}$ at $T=0.01 \mathrm{~K}$ for a tube of length $L=800 \mathrm{~nm}$ and $R=1.5 \mathrm{~nm}[3]$. This indicates that, at low temperatures, four-phonon decays give only a small contribution to the observed losses, which are $Q \approx 10^{4}$ at $T=1 \mathrm{~K}$ and $Q \approx 10^{5}$ at $T=0.01 \mathrm{~K}$ [3]. Although small, these losses are still much greater than the three-phonon contributions: $Q_{3}=3.1 \times 10^{27}$ at $1 \mathrm{~K}$ and even greater at $0.01 \mathrm{~K}$. Our analysis, contrary to the aforementioned theoretical studies, suggests that the losses observed by Hüttel et al. [3] are mostly extrinsic.

J.L. supported by DOE \# DE-FG02-07ER46432.

[1] B. Lassagne, Y. Tarakanov, J. Kinaret, D. GarciaSanchez, and A. Bachtold, Science 325, 1107 (2009).

[2] G. Steele, A. Huettel, B. Witkamp, M. Poot, H. Meerwaldt, L. Kouwenhoven, and H. van der Zant, Science 325, 1103 (2009).

[3] A. Huettel, G. Steele, B. Witkamp, M. Poot, L. Kouwenhoven, and H. van der Zant, Nano Lett. 9, 2547 (2009).

[4] K. Jensen, K. Kim, and A. Zettl, Nat. Nanotechnol. 3, 533 (2008).

[5] B. Lassagne, D. Garcia-Sanchez, A. Aguasca, and A. Bachtold, Nano Lett. 8, 3735 (2008).

[6] H.-Y. Chiu, P. Hung, H. Postma, and M. Bockrath, Nano Lett. 8, 4342 (2008).

[7] B. Witkamp, M. Poot, and H. van der Zant, Nano Lett. 6, 2904 (2006).

[8] H. Jiang, M.-F. Yu, B. Liu, and Y. Huang, Phys. Rev. Lett. 93, 185501 (2004).

[9] S. P. Hepplestone and G. P. Srivastava, Phys. Rev. B 74, 165420 (2006).

[10] A. D. Martino, R. Egger, and A. O. Gogolin, Phys. Rev. B 79, 205408 (2009).

[11] X. Wei, Q. Chen, S. Xu, L. Peng, and J. Zuo, Adv. Funct. Mater. 19, 1753 (2009).

[12] H. Suzuura and T. Ando, Phys. Rev. B 65, 235412 (2002).

[13] W. Kohn and L. Sham, Phys. Rev. 140, A1133 (1965).

[14] K. V. Zakharchenko, M. I. Katsnelson, and A. Fasolino, Phys. Rev. Lett. 102, 046808 (2009).

[15] D. Sánchez-Portal, E. Artacho, J. M. Soler, A. Rubio, and P. Ordejón, Phys. Rev. B 59, 12678 (1999).

[16] D. Donadio and G. Galli, Phys. Rev. Lett. 99, 255502 (2007).

[17] D. Song, F. Wang, G. Dukovic, M. Zheng, E. Semke, L. Brus, and T. Heinz, Phys. Rev. Lett. 100, 225503 (2008).

[18] S. Sapmaz, Y. Blanter, L. Gurevich, and H. van der Zant, Phys. Rev. B 67, 235414 (2003).

[19] P. Zhang, P. Lammert, and V. Crespi, Phys. Rev. Lett. 81, 5346 (1998).

[20] B. Perrin, Phys. Rev. B 36, 4706 (1987). 